\title{
The Use of a New Clinical Reasoning Framework to Facilitate The Assessment and Management of Temporomandibular Disorders
}

Alexander Weden*

Department of Maxillofacial, Queens Medical Centre, Nottingham University Hospitals, Derby Road, Nottingham, NG7 2UH, UK Department of Physiotherapy, Physiotherapy Outpatients, Queens Medical Centre, Nottingham University Hospitals, Derby Road, Nottingham, NG7 2UH, UK

*Corresponding author: Alexander Weden, Department of Maxillofacial, Queens Medical Centre, Nottingham University Hospitals, Derby Road, Nottingham, NG7 2UH, UK

Received date: 19 February, 2022 |

Accepted date: 28 February, 2022

Published date: 2 March, 2022

Citation: Weden A. (2022) The Use of a New Clinical Reasoning Framework to Facilitate The Assessment and Management of Temporomandibular Disorders. J Dent Oral Epidemiol 2(1): doi https://doi.org/10.54289/JDOE2200101

Copyright: () 2022 Weden A. This is an open-access article distributed under the terms of the Creative Commons Attribution License, which permits unrestricted use, distribution, and reproduction in any medium, provided the original author and source are credited.

\begin{abstract}
Background: Temporomandibular Disorders (TMDs) are the most common form of non-odontogenic orofacial pain. TMDs are considered a biopsychosocial disorder with a multifactorial pathogenesis. Patients with TMDs frequently present with cervical spine disorders, headaches and otological complaints. Physiotherapists are often sought to assess and treat TMDs. The use of a radar graph and triangulation to represent phenotyping patients with complex pain presentations has been discussed in the literature with the aim of supporting the best course of treatment for patients with complex and enigmatic pain presentations.

Objective: This paper presents the adaptation and development of the radar graph model for the assessment and management of TMDs. It was developed in a Maxillofacial service in the United Kingdom and has been used to support the assessment and physiotherapeutic management of patients with a TMD since 2019. Herein we describe the different domains we prioritise in the assessment and the means by which they are assessed. It is hoped by presenting our radar graph in the literature other clinicians may find it helpful or adapt it as they see fit. For illustrative purposes, we also describe two case studies of patients who underwent assessment and treatment with us using the radar graph model.

Conclusion: Assessing and treating complex conditions such as TMD poses many challenges. The radar model discussed has been of value in our service and we believe this paper demonstrates its adaptability to TMDs. Evaluation of its ability to augment treatment planning and delivery is required, however.
\end{abstract}

Keywords: Temporomandibular disorders, Headache, Cervical spine, Physiotherapy, Clinical reasoning

\section{Introduction}

Temporomandibular disorders (TMD) refer to pain and dysfunction manifesting from the masticatory myofascial and temporomandibular joint [1]. The incidence of TMDs is estimated to be $5-10 \%$ of the populations studied and inclined to affect females in their 'middle years' predominantly [2]. TMD symptomology can lead to considerable compromise of quality of life for those chronically affected and impart 
significant healthcare costs [3]. TMDs are the main cause of non-odontogenic orofacial pain whose pathogenesis is now widely accepted as biopsychosocial and multifactorial [4]. TMDs are responsive to musculoskeletal physiotherapeutic interventions such as exercise and manual therapies, however the effect size of such approaches in isolation is variable which likely reflects the multifactorial and biopsychosocial nature of TMDs [5].

Those experiencing a TMD often present with concomitant cervical spine disorders, primary headaches and otological symptoms [6-8]. The exact causal relationship between the cervical spine disorders, TMDs and primary headaches remains speculative, but treatment of the cervical spine can improve symptoms of TMDs [9] and can attenuate primary headaches [10]. The otological symptoms that frequently accompany a TMD are otalgia, tinnitus, aural fullness and dizziness. The pathogenesis of these symptoms and the nature of their relationship to TMDs is largely conjectural however evidence suggests treatment of the orofacial complex [11] and cervical spine via musculoskeletal therapies can also attenuate these symptoms [12].

The majority of patients with a chronic TMD suffer from a combination of a TMD, cervical spine disorders, headaches and otological symptoms [6-8]. In light of this, musculoskeletal clinicians treating TMDs would be expected to assess and treat what is probably best considered as the cranio-cervical-mandibular complex rather than just the masticatory system and will therefore have a number of diagnoses to consider. TMDs are precipitated by and malleable to the broad array of factors that cause and influence most pain conditions such as genetics, somatisation, psychopathology, central sensitisation, and lifestyle factors [13]; therefore, the consideration of multiple body systems beyond the musculoskeletal system (but not to the exclusion of it) should be undertaken in the evaluation of patients with a diagnosis of TMD. Given the potential complexity of patients reporting a TMD and the contemporary mandate to deliver care that is personalised [14], an assessment framework that evaluates both musculoskeletal and nonmusculoskeletal factors that can cause and influence TMD symptomology could be of significant help for clinically reasoning the unique needs of the presenting patient with a TMD and its accompanying conditions.

The use of a Radar Graph to represent the outcomes of phenotyping patients with pain in a multi-dimensional way was proposed by [15]. The development of the radar graph tool was not only a response to the evidentiary and philosophical limitations of subgrouping patients with musculoskeletal pain, but as a reflection of the need to have a tool that would potentially offer more clarity to the 'driver' or 'drivers' of a patient's pain respecting its multidimensionality. [15] were enthusiastic for the principle of the radar graph to be adapted across varying patient populations and professional disciplines. As a response to this encouragement and the absence of such a framework in the literature specific to TMDs, an assessment framework underpinned by the radar graph concept to phenotype patients with a diagnosis of TMD attending a specialist Maxillofacial service in Nottingham was developed by the author and has subsequently been utilised to inform the management of approximately 150 patients.

This paper has two aims. Firstly, it is an opportunity for the author to share this radar graph for clinicians treating TMD. Clinicians are welcome to use the radar graph if they deem it of sufficient value and adapt and improve it as they see fit. Then for illustrative purposes, we will present the clinical history, radar graphs, treatment strategies and outcomes of two patients who underwent phenotyping and then treatment informed by their radar graphs.

\section{The Radar Graph}

The primary purpose of this_multidimensional assessment tool is to capture and represent the degree of complexity of the patient and to understand the relative degree that each domain and its related phenomena contribute to the experience of the patient with a TMD. Such information can then provide insight as to when, for example, an integrative and holistic management package (requiring referral to services such as Neurology or Psychology) is needed or when a pathway underpinned by musculoskeletal modalities would most likely be appropriate.

The radar graph presented here has seven domains. This is not intended to mirror the same number of domains described in the [15], but as a reflection of this author's beliefs that the 
following seven domains are important and clinically deliverable for the evaluation of patients with a TMD. As will be observed, some domains used will be relevant to all musculoskeletal pain, whilst the remaining domains pertain to assessing patients with TMD and its accompanying conditions. When searching relevant measurement tools, many provided results that would quantify the phenomena they were measuring as mild, moderate, severe and extreme allowing a sense of the magnitude of influence on the patients' symptoms. When considering the means by which the phenomena relating to the domain was to be assessed (i.e., physical assessment versus questionnaire) the author considered:

- The evidence base supporting the target factors as important contributors to TMD

- The empirical robustness of the assessment approach

- The pragmatic application in the clinical setting

- Consideration of patient burden

- Consideration of tools that can serve both assessment and outcomes roles

In our clinical practice the data to construct the radar graph begins after assessment by the Maxillofacial consultant. Once a diagnosis of a TMD is established Physiotherapy is offered as an option for treatment. If the patient consents to a trial of physiotherapy, they are asked to attend an appointment with the author having filled in the 5 questionnaires that will be discussed shortly. In the appointment with the author a detailed subjective and objective exam is undertaken. The findings from this appointment and the results from the questionnaires are aggregated to generate the radar graph. The radar graph then provides a basis from which a dialogue can commence to establish an agreed management strategy with the patient in keeping with a shared decision-making model of care [16]. The domains and the means by which they are assessed are described here in no particular order.

Psychological Wellbeing (Depression Anxiety Stress Scale-21)

The bi-directionality of pain and negative psychological phenomena is well established and critical in the evaluation of any patient with pain [17]. The comorbidity of TMD, headaches and adverse mental health is well established $[18,19]$ and psychological therapies on their own or in combination with physical therapies can attenuate pain in patients with TMD [20] and headache [21]. Identifying those who would benefit from psychological therapy in isolation or as an adjunct in their package of care is important for management optimisation.

The DASS-21 is a self-report questionnaire to measure three related negative emotional states of Depression, Anxiety and Stress. It has adequate evidence of validity and reliability for use in musculoskeletal pain conditions [22] though it does not replace formal assessment and diagnosis by a trained psychotherapist. However, it does provide an estimate of the severity of negative mood states reported by the patient and has shown value as a screening tool for depressive and anxiety disorders [23]. In practice we have found it useful for facilitating dialogue around the importance of psychological well-being and its intimate relationship to TMDs and indeed all pain conditions.

\section{Central Sensitisation (Central Sensitisation Inventory)}

This term refers to nociplastic change of central nervous system pathways and is implicated in generating, amplifying, and maintaining painful conditions including TMD and headaches [24]. Such changes may influence the patients' response to physical stimuli, explain ancillary symptoms to pain such as photo or phonophobia, and affect management strategies and prognosis [25]. The Central Sensitisation Inventory is a patient administered questionnaire intended to capture a range of experiences that are thought to indicate such central nociplastic change [26]. The CSI has established grades of severity ascribed to scores from 0 -100 reflecting the spectrum of subclinical to extreme sensitisation.

\section{Headache Severity (Headache Impact Test 6)}

When present, we prioritize establishing a diagnosis for headache via the ICHD-3b criteria if one is not already established. Cognisant that headache can worsen TMD symptomology [27], establishing the severity of its presence offers the physiotherapist the opportunity to discuss and initiate headache management strategies such as manual therapy, acupuncture or referral for psychotherapeutic or Neurology services.

The HIT-6 has strong evidence of validity and reliability in assessing headache impact and has good applicability to all headache types [28]. It is quick for the patient to fill in and 
has five progressive dimensional categories for severity of impact from no impact on quality of life to very severe impact. Not only does the HIT-6 offer the aforementioned opportunities but it can also serve as a patient-reported outcome measure (PROM) for evaluation of change over time.

\section{Sensorimotor Disintegration (Dizziness Handicap} Inventory)

The presence of dizziness with patients suffering from TMD and headaches is very common [28], though causation has not been established. Dizziness has been identified as a manifestation of various neuro-otological disorders [29], however the critical integration of afferents from dense proprioceptors in the upper cervical spine into numerous vestibular and oculo-motor reflexes [30] and the high coexistence of upper cervical spine dysfunctions in people with TMD [31] and headache [33] provides a compelling rationale for musculoskeletal physiotherapists to further explore such symptoms.

Prior research has indicated excellent reliability and strong evidence of validity in measuring severity of dizziness [33] and therefore provides us with insight into the degree dizziness contributes to the patient's experience. It also has value in screening for cervicogenic dizziness [34] thereby helping to identify those whose dizziness symptoms might benefit from treatment of the upper cervical spine or might benefit from a referral to an Ear, Nose and Throat specialist. It also provides a robust measure of response to treatment and therefore can double-up as another evaluative PROM.

The DHI has established cut off scores for mild (16-34), moderate (36-52) and severe handicap (54+). For consistency with the other tools used, we dividing the scores of 54-100 (maximal score) in two and assigned the label of extreme to a score of $75 / 100$ or over.

\section{Temporomandibular Dysfunction (Jaw Functional}

\section{Limitation Scale-8)}

A disease and organ specific measure of masticatory function and limitation is an important component of information in the radar graph for diagnostic and evaluatory purposes. It is intended to quantify the level of dysfunction of the masticatory system. The Jaw Function Limitation Scales 20 and 8 offer tools for assessing the functional status of the masticatory system [35] and are embedded in Axis II of the Diagnostic Criteria for Temporomandibular Disorders (DC/TMD) [36]. Balancing the need for detail without burdening patients, the JFLS- 8 was preferred over the JFLS20 due to brevity with comparably good psychometric properties.

To date there are no empirically defined descriptors of dysfunction assigned to the scores of 0-80 for the JFLS-8. In the absence of such information, we assigned levels of severity to the following scores: 11-30 (mild), 31-50 (moderate), 51-70 (severe) \& 71-80 (extreme). These severity delineations can be reconsidered when appropriate empirical work is published.

\section{Cervical Spine Dysfunction}

Given the therapeutic opportunity in treating the cervical spine to improve TMD and headaches we felt it was essential this was included $[\mathbf{8 , 9}]$. The cluster of tests described below have been extensively used for assessment of cervical spine dysfunction in various studies relating to cervical spine disorders, TMDs and Headaches [37-40]. We evaluate cervical spine dysfunction through direct observation and manual assessment. Binary answers such as positive/negative or present/absent are used to score on the domain's continuum:

- Cervical range of movement both globally and during the Cervical Flexion/Rotation test (Limitation evident present/absent)

- Arthrogenic hypomobility at the Atlano-Occipital joint to (and including) the $\mathrm{C} 2-3$ facets to discern hypomobility of Passive Physiological Intervertebral Movements (PPIVMs). (Manual assessmentpresent/absent)

- Myofascial trigger points known to refer to the orofacial and headache regions as per Axis I of the DC/TMD [36] \& International Delphi consensus guidance [36,40] (manual assessment- present/absent)

- Neuromuscular weakness of the cervical spine (biopressure feedback test-present/absent)

This phase of assessment also allows the inclusion of the Adapted Spurlings Test with the Manual Rotation Test to support differential diagnosis of Cervical Somatic Tinnitus for those patients' reporting tinnitus as an otological symptom 
[41]. The score for cervical spine dysfunction progresses along the continuum of mild to extreme based on the number of impairments found.

\section{General Health Status \& Lifestyle Assessment}

Lifestyle and general health factors are discussed in the subjective examination. Factors of interest are: sleep, physical activity levels, BMI, and smoking. These specific factors are investigated as all are implicated in precipitating and influencing pain disorders, are easy to discern and are modifiable factors with appropriate education and behavioural change with the view to imparting pain modulation [42-45]. In this domain, as within the spinal domain, binary answers of yes or no are used to determine position along the domain's continuum from absent to extreme.

Sleep - The patient is asked whether they achieve appropriate health inducing volumes of sleep per night as would be expected for their age [46] (yes/no).

Smoking - Does the patient currently smoke smoke (Yes/no)

Body Mass Index (BMI) - Is the patient within their expected BMI (yes/no)

Exercise - Do they meet national recommended activity levels of exercise [47] (yes/no)

\section{Case studies}

To provide a concrete illustration of how these tools are used and interpreted, below are 2 case studies presenting examples of actual patient cases for which radar graphs were generated

\section{Case study 1}

Jane is a 58-year-old Caucasian female referred by her medical general practitioner for her bilateral TMD. Her symptoms started 2 years prior to attending the Maxillofacial service with no known etiology. Management prior to her referral was with her dentist via the provision of a bite raising appliance, gentle massage of her masseters and a soft food diet. Jane has a diagnosis of fibromyalgia, irritable bowel \& bladder syndromes, functional dyspepsia, and a history of migraines. She has experienced bouts of a mixture of generalised anxiety disorder and depression since she was a teenager. Her medications are Citalopram, Co-codemol \& Omeprazole. She was not working at the time due to her illhealth.
Her presenting symptoms were bilateral myofascial pain (with referral) of the masticatory apparatus, bilateral TMJ arthralgia (DC/TMD) and reduced oral opening (Maximal Mouth Opening of $15 \mathrm{~mm}$ due to pain and fear of movement as reported by the patient). Jane's pain exacerbated with eating, talking, yawning and in cold weather and modest pain improvement was via the application of a hot towel to her face and by resisting moving her mandible. Jane was prone to bruxism both by day and by night. She also suffered from daily headaches in the form of tension type headaches accompanied by nausea and photophobia. She experienced intermittent aural fullness, otalgia, and disequilibrium and constant tinnitus. Jane's mental health had recently worsened prior to our consultation such that she stated she was suffering from a bout of depression again. Her sleep was poor commensurate with her FMS whereby she achieved a broken 5-6 hours per night on average. She did not partake in any exercise, but she was a non-smoker and her BMI scored her as underweight.

On clinical examination her cervical rotation was limited to half of expected ROM. Her upper fibres of trapezius and sternocleidomastoids appeared hypertrophic and referred pain to her face and forehead on palpation, while palpation of obliquus capitis superior reproduced her headache. Manual assessment of her upper cervical spine demonstrated hypomobility of her atlantoaxial and atlanto-occipital joint bilaterally. The cervical flexion/rotation test was tight and sore but around 40 degrees in each direction were achieved. She struggled to manage to hold 10 seconds at $24 \mathrm{mmhg}$ on the craniocervical flexion test, partly due to weakness and partly due to increased discomfort in her suboccipitals when trying the test. Her masseters were highly sensitised such that they were nearly allodynic to touch. Intra-oral examination of masseter was not possible due to her limited and painful opening but palpation of her TMJ was possible and reproduced some of her TMD pain.

Jane reported her cranio-facial pain averaged $7 / 10$ over the preceding week. Jane expressed her goals from treatment to be for her to be able to open her mouth with less pain so eating would be more comfortable, her headaches to be episodic rather than constant and for her neck to be less restricted in its movement. 


\section{Treatment}

Jane's history and clinical findings indicated multiple issues likely driving her pain experience, and these were visualized by plotting them on a radar graph (Figure xx). Accordingly, an approach of multiple modalities seemed likely to impart therapeutic gains, and the radar graph facilitated open communication between Jane and us towards a shared understanding of her pain and most relevant treatment targets. The beginning of Jane's course of treatment included extensive time educating her on the nature and complex biopsychological pathophysiology of Fibromyalgia, TMDs and current pain neuroscience. Embedded within this multimodal intervention plan was discussion and education about gastrointestinal health and pain, sleep hygiene, the effect of mood on pain, finding meaningful physical activity and a course of acupuncture and dry needling as a symptom management strategy prior to the introduction of manual and exercise therapy that Jane was eventually taught to selfadminister. Treatment occurred in 6 visits over 12 weeks.

Jane's pain per week score was reduced from $7 / 10$ on the first visit to 2/10 after 12 weeks. Her JLF-8 score improved from 50 to 5 and her maximal mouth opening score improved from $18 \mathrm{~mm}$ to $35 \mathrm{~mm}$. The HIT- 6 score reduced from 66 to 50 (extreme to moderate impact). Jane sought out a dietician and found the Low FODMAP diet helpful in reducing her IBS symptoms. Jane reported an improvement in mood both due to the support of her psychotherapist, improved IBS symptoms and due to the self-efficacy, she now had over her cranio-facial pain.

\section{Case study 1:}

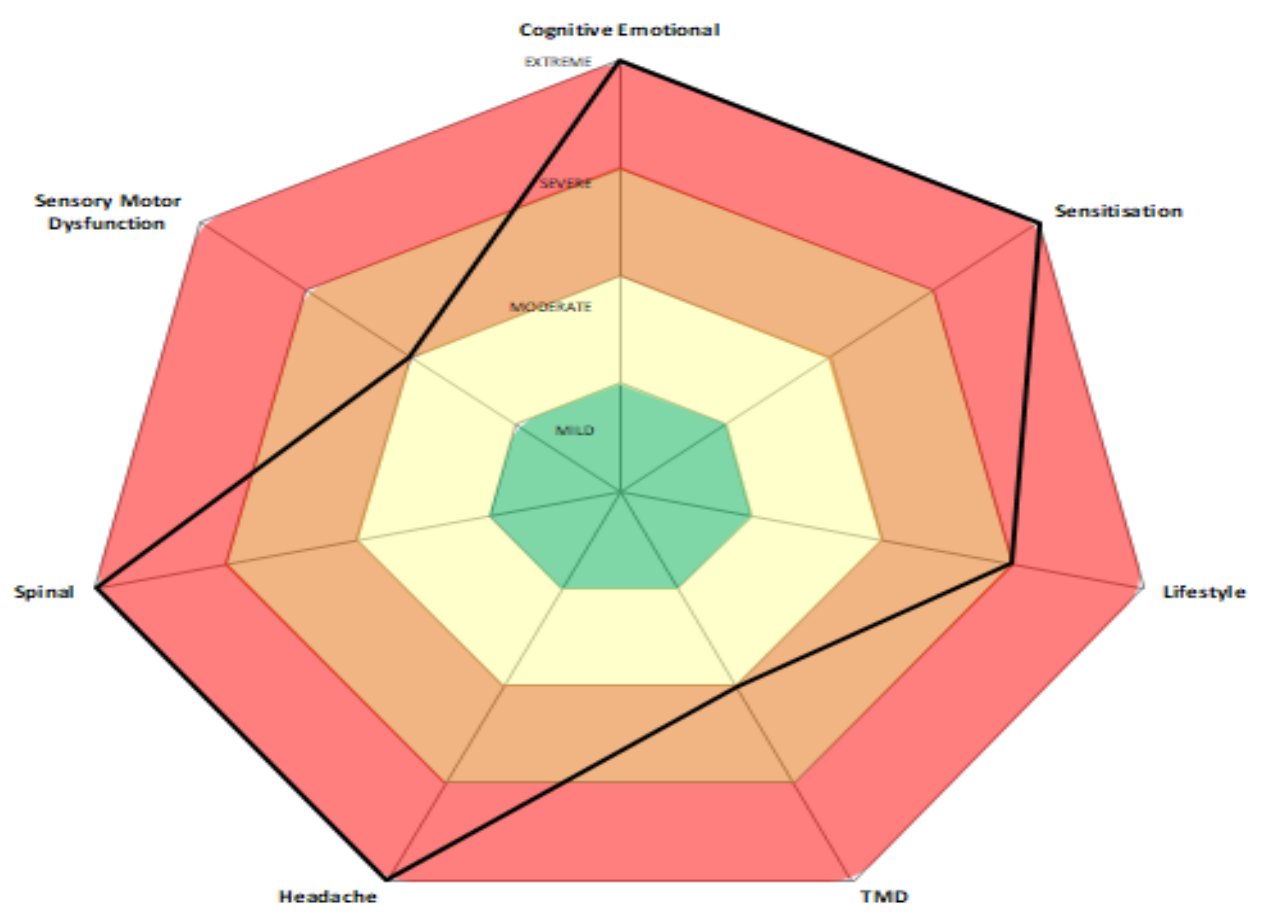

\begin{tabular}{|l|l|l|}
\hline Domain & Score & Level \\
\hline Psychological (DASS-21) & D-14, A- 11, S -6 & Extreme (D \& A) \\
\hline Central Sensitisation (CSI) & $77 / 100$ & Extreme \\
\hline Jaw Function Limitation (JFLS-8) & 50 & Moderate \\
\hline Sensory motor (DHI) & 50 & Extreme \\
\hline Headache (HIT-6) & 66 & Extreme \\
\hline Spinal Dysfunctions & $4 / 4$ & Severe \\
\hline Lifestyle & $3 / 4$ & \\
\hline
\end{tabular}




\section{Case study 2}

Sharon is a 36 -year-old mother of 3 referred by her medical general practitioner. Sharon had a 20-year history of a painless 'clicky' right jaw that progressively worsened to a jaw that would produce a painful click and occasional locking and myalgia of her right masseter. These symptoms are provoked by extended periods of talking or laughing. Sharon denied any day or night time bruxism. She denied any constant cervical spine symptoms other than some restriction of range and 'achyness' during her migraines. She also denied any otological symptoms. Sharon explained she has been significantly troubled with right sided headaches since she was a teenager that had been diagnosed as episodic migraines by her GP. Her headaches presented as an arrestingly severe right sided headache in the periocular region accompanied by nausea and photophobia. These headaches which would fit ICDH-3 diagnostic criteria for migraine and would occur on average twice a month with no clear precipitating factors. When not experiencing her migraine, Sharon reported a milder right sided headache that would start from her periocular area back over most of her temporal region around 3 days per week. She was otherwise fit and well with no medical history to speak of. Her medications were propranolol and sumatripton for her migraines and the contraceptive pill. Her sleep was a little erratic as the mother of 3 young children such that she would achieve maybe 7 hours a night at best. She would exercise most days, had a BMI of 20 and was a non-smoker.

On clinical examination Sharon's right scapula was markedly lower than her left. She was very inclined to sit in a slumped posture with a forward head position. Cervical spine rotation to the left was full but to the right was limited to two thirds of normal ROM. She had active myofascial trigger points in her right upper fibres of trapezius, SCM, obliquus capitis superior (which reproduced her background headache) and obliquus capitis inferior. Sharon had hypomobile facets at her right $\mathrm{C} 1 / 2$ and $\mathrm{C} 2 / 3$ and her atlas felt rotated to the right. She managed to achieve $26 \mathrm{mmhg}$ three times before commenting on it being effortful during the cranio-cervical flexion test. Oral opening was $23 \mathrm{~mm}$ with subtle deviation of the mandible to the right and an absence of protrusion and upper cervical extension. Her right TMJ was tender to touch and symptoms suggestive of Anterior Disc Displacement with Reduction was palpable also during oral opening. Her right Masseter was also hypertrophic compared to her left.

Sharon reported the combination of her right TMD pain and periocular headache to average 6/10 per week. When discussing her objectives for treatment she stated she would hope for a $50 \%$ reduction in her headache frequency and an improvement on maximal mouth opening.

\section{Case study 2:}

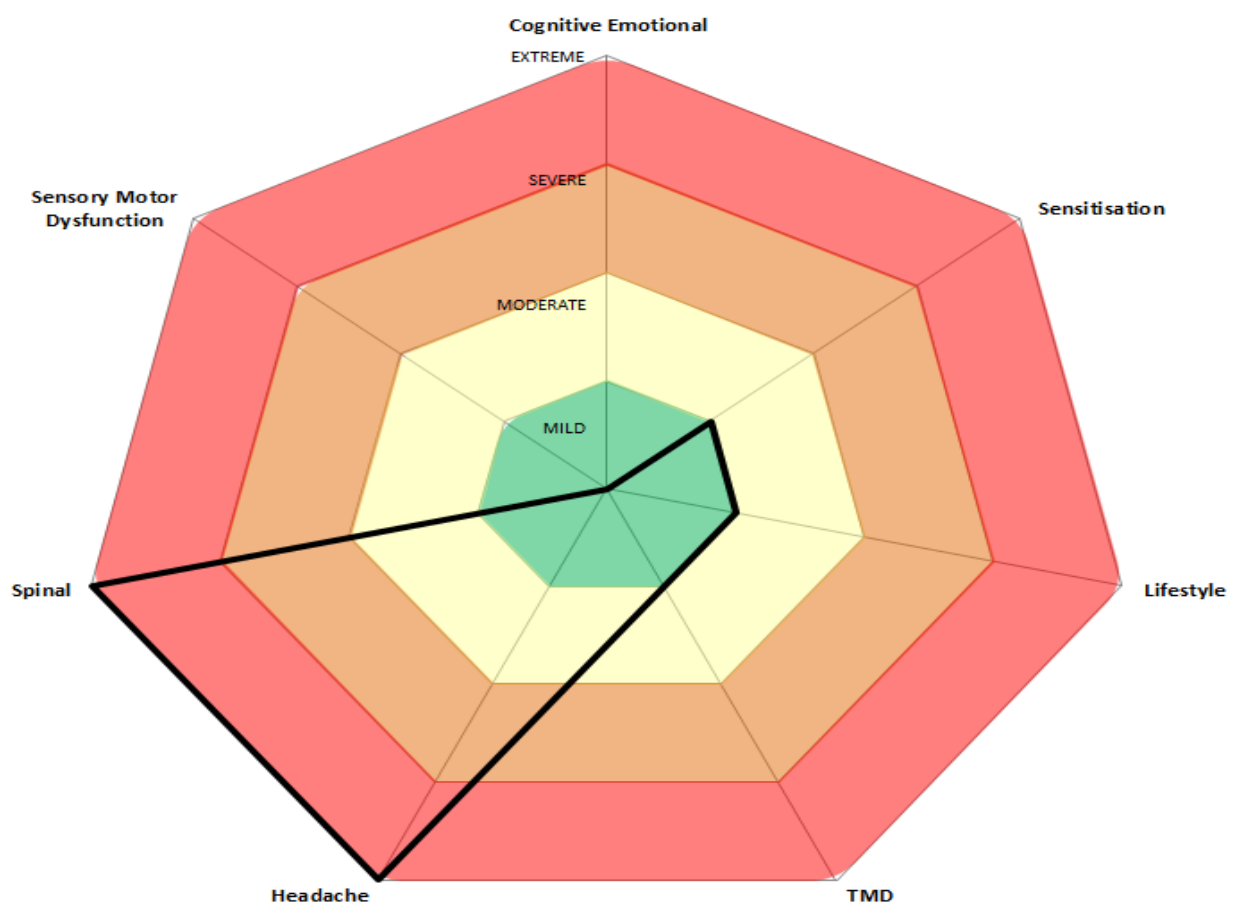




\begin{tabular}{|l|l|l|}
\hline Domain & Score & Level \\
\hline Psychological (DASS-21) & D-1, A- 1, S -4 & Normal \\
\hline Central Sensitisation (CSI) & $30 / 100$ & Mild \\
\hline Jaw Function Limitation (JFLS-8) & 26 & Mild \\
\hline Sensory motor (DHI) & 10 & Subclinical \\
\hline Headache (HIT-6) & 62 & Extreme \\
\hline Spinal Dysfunctions & $4 / 4$ & Extreme \\
\hline Lifestyle & $1 / 4$ & Mild \\
\hline
\end{tabular}

\section{Treatment}

Sharon's radar graph did not demonstrate numerous significant drivers to her headaches and TMD. Instead, a couple of mild contributing factors were noted and the likely dominant driver in the form of her cervical spine dysfunction was highlighted. Therefore, after a discussion around the multifactorial pathophysiology of migraine, Sharon agreed to a course of physiotherapy aimed at addressing her cervical spine dysfunctions and TMD in the form of manual therapy and exercise to her upper cervical spine and masticatory myofascial. This was also complimented by some improvement in sleep hygiene and subtle dietary changes in keeping with SEEDS Lifestyle management strategies [48].

\section{Outcome}

After 5 sessions and 10 weeks of physiotherapy Sharon's average pain per week for her cranio-facial pain had reduced from $6 / 10$ to $2 / 10$. Her HIT-6 score had reduced from $62-52$ (Extreme to moderate), she reported her background headache incidence had reduced by half and she had not had any of her migraines in the preceding two months. The JFLS8 scores improved from 26 to 14 and maximal mouth opening had improved from $23 \mathrm{~mm}$ to $35 \mathrm{~mm}$ with a 'subtle click'.

\section{Discussion}

The purpose of this manuscript was to describe the development and use of a radar graph as a tool for data visualization to facilitate sense-making of a complex clinical condition like TMDs. It is hoped this paper has provided an insight and overview of the potential complexity of managing TMDs from the perspective of a clinician embedded within the field and rationalized the need for a clinical reasoning framework that is reflective of the multidimensional nature of the condition. To date the use of such a framework remains untested in its ability to optimise patient care, but the philosophical motives to capture the multiple factors that can influence pain is consistent with the current multidimensional understanding of pain and TMDs. We hope this paper has exemplified the adaptability and utility of the radar graph concept in general and as it can relate to assessing TMDs and their associated conditions. As demonstrated in the second case study, we believe this graph can also support therapists working alongside patients with a primary complaint of headache given the common factors that influence both conditions.

Despite the theoretical strengths of the framework presented we are cognisant of the limits of what is (or is not) captured. It was always the hope that the domains included are comprehensive in respect to etiological and driving factors of TMD, however some factors such as oral parafunction, breathing pattern disorders, somatization, or psychological constructs such as catastrophization are not captured. Perhaps therefore it's best to see the included domains as valuable for most patients but not fully comprehensive for all patients and so therapists who choose to use this radar graph must not have their clinical reasoning limited by the domains included. That said, reassuringly two recent international Delphi studies on the most useful assessments for physiotherapists to use in assessing headaches [39] and TMD [38] would find most (if not all) tests described to construct our radar graphs consistent with their consensus-based recommendations. From the patient perspective, many of our patients have stated how they valued the multidimensional nature and depth of the assessment process described, especially when the discourse includes education relating to the evidence and biological 
rational connecting pain, mental health and systemic and metabolic wellbeing. To date, when asked by us, no patients have stated that our assessment process to construct their radar graphs has felt unnecessarily burdensome. Many have also commented how representing their cranio-mandibular complaints in a diagram circular in nature cultivates a sense of a 'holistic' process having been undertaken, thereby reflecting the biopsychosocial nature of their condition.

\section{Conclusion}

The use of the radar graph has been of notable value for the purposes of clinical reasoning and early identification of those patients needing unimodal or blended approaches to their management for our service in Nottingham. The breadth and depth of information gained and represented in the radar graph feels like a meaningful step forward on the journey to trying to phenotype patients struggling with a TMD. It is our experience however that the radar graph delivers the most value when not used algorithmically, but when combined with the rich information drawn from the qualitative pain narrative of the patient with the intent of delivering compassion-based care [49] for an optimal therapeutic alliance between therapist and patient. That said, we recognise this system remains untested in its ability to deliver better patient experiences and outcomes so clinicians should be mindful of this should they consider using it.

Disclosure statement: The author reports no conflicts of interest

Acknowledgements: Thank you to Professor David Walton for his invaluable support and guidance with this manuscript. Thank you also to Drs Vicky Booth and Katie Robinson for their encouragement and support to write this manuscript. The author did not receive any grants or financial support for this manuscript. The author declares no conflicts of interest.

\section{Bibliography}

1. Kapos FP, Exposto FG, Oyarzo JF, Durham J. (2020) Temporomandibular disorders: a review of current concepts in aetiology, diagnosis, and management. Oral Surgery. 13.
2. Akhter R. (2019) Epidemiology of Temporomandibular Disorder in the General Population: A Systematic Review. Advances in Dentistry \& Oral Health.10(3).

3. Durham J, Shen J, Breckons M, et al. (2016) Healthcare Cost and Impact of Persistent Orofacial Pain. Journal of Dental Research. 95(10): 1147-1154.

4. Zakrzewska JM. (2013) Multi-dimensionality of chronic pain of the oral cavity and face. The Journal of Headache and Pain. 14(1): 37.

5. Armijo-Olivo S, Pitance L, Singh V, Neto F, Thie N, et al. (2015) Effectiveness of Manual Therapy and Therapeutic Exercise for Temporomandibular Disorders: Systematic Review and Meta-Analysis. Physical Therapy. 96(1): 9-25.

6. Cuenca-Martínez F, Herranz-Gómez A, MadroñeroMiguel B, et al. (2020) Craniocervical and Cervical Spine Features of Patients with Temporomandibular Disorders: A Systematic Review and Meta-Analysis of Observational Studies. Journal of Clinical Medicine. 9(9): 2806.

7. Franco AL, Gonçalves DAG, Castanharo SM, Speciali JG, Bigal ME, et al. (2010) Migraine is the most prevalent primary headache in individuals with temporomandibular disorders. Journal of Orofacial Pain. 24(3): 287-292. Accessed February 5, 2021.

8. La Touche R, Martínez García S, Serrano García B, et al. (2020) Effect of Manual Therapy and Therapeutic Exercise Applied to the Cervical Region on Pain and Pressure Pain Sensitivity in Patients with Temporomandibular Disorders: A Systematic Review and Meta-analysis. Pain Medicine. Published online.

9. Fernández-de-las-Peñas C, Cuadrado ML. (2016) Physical therapy for headaches. Cephalalgia. 36(12): 1134-1142.

10. Stechman-Neto J, Porporatti AL, Porto de Toledo I, et al. (2016) Effect of temporomandibular disorder therapy on otologic signs and symptoms: a systematic review. Journal of Oral Rehabilitation. 43(6): 468-479.

11. Michiels S, Naessens S, Van de Heyning P, et al. (2016) The Effect of Physical Therapy Treatment in Patients with Subjective Tinnitus: A Systematic Review. Frontiers in Neuroscience. 10. 
12. Slade GD, Fillingim RB, Sanders AE, et al. (2013) Summary of Findings from the OPPERA Prospective Cohort Study of Incidence of First-Onset Temporomandibular Disorder: Implications and Future Directions. The Journal of Pain. 14(12): T116-T124.

13. Cutica I, Vie GM, Pravettoni G. (2014) Personalised medicine: The cognitive side of patients. European Journal of Internal Medicine. 25(8): 685-688.

14. Edwards RR, Dworkin RH, Sullivan MD, Turk DC, Wasan AD. (2016) The Role of Psychosocial Processes in the Development and Maintenance of Chronic Pain. The Journal of Pain.17(9): T70-T92.

15. Walton DM, Elliott JM. (2018) A new clinical model for facilitating the development of pattern recognition skills in clinical pain assessment. Musculoskeletal Science and Practice. 36: 17-24.

16. Hoffmann TC, Lewis J, Maher CG. (2020) Shared decision making should be an integral part of physiotherapy practice. Physiotherapy. 107: 43-49.

17. Fillingim RB, Ohrbach R, Greenspan JD, et al. (2013) Psychological Factors Associated with Development of TMD: The OPPERA Prospective Cohort Study. The Journal of Pain. 14(12): T75-T90.

18. Probyn K, Bowers H, Caldwell F, et al. (2017) Prognostic factors for chronic headache: A systematic review. Neurology. 89(3): 291-301.

19. Litt MD, Shafer DM, Kreutzer DL. (2010) Brief cognitive-behavioural treatment for TMD pain: Longterm outcomes and moderators of treatment. Pain. 151(1): 110-116.

20. Lee HJ, Lee JH, Cho EY, Kim SM, Yoon S. (2019) Efficacy of psychological treatment for headache disorder: a systematic review and meta-analysis. The Journal of Headache and Pain. 20(1).

21. Parkitny L, McAuley JH, Walton D, et al. (2012) Rasch analysis supports the use of the Depression, Anxiety, and Stress Scales to measure mood in groups but not in individuals with chronic low back pain. Journal of Clinical Epidemiology. 65(2): 189-198.

22. Ng F, Trauer T, Dodd S, Callaly T, Campbell S, Berk M. (2007) The validity of the 21 -item version of the Depression Anxiety Stress Scales as a routine clinical outcome measure. Acta Neuropsychiatrica. 19(5): 304310.

23. Garrigós-Pedrón M, La Touche R, Navarro- Desentre P, Gracia-Naya M, Segura-Ortí E. (2019) Widespread mechanical pain hypersensitivity in patients with chronic migraine and temporomandibular disorders: relationship and correlation between psychological and sensorimotor variables. Acta Odontologica Scandinavica. 77(3): 224231.

24. Woolf CJ. (2011) Central sensitization: Implications for the diagnosis and treatment of pain. Pain. 152(Supplement): S2-S15.

25. Scerbo T, Colasurdo J, Dunn S, Unger J, Nijs J, et al. (2017) Measurement Properties of the Central Sensitization Inventory: A Systematic Review. Pain Practice. 18(4): 544-554.

26. Costa Y, Alves da Costa D, de Lima Ferreira A, et al. (2017) Headache Exacerbates Pain Characteristics in Temporomandibular Disorders. Journal of Oral \& Facial Pain and Headache. 31(4): 339-345.

27. Haywood KL, Mars TS, Potter R, Patel S, Matharu M, Underwood M. (2017) Assessing the impact of headaches and the outcomes of treatment: A systematic review of patient-reported outcome measures (PROMs). Cephalalgia. 38(7): 1374-1386.

28. Porto De Toledo I, Stefani FM, Porporatti AL, et al. (2016) Prevalence of otologic signs and symptoms in adult patients with temporomandibular disorders: a systematic review and meta-analysis. Clinical Oral Investigations. 21(2): 597-605.

29. Chan Y. (2009) Differential diagnosis of dizziness. Current Opinion in Otolaryngology \& Head and Neck Surgery. 17(3): 200-203.

30. Devaraja K. (2018) Approach to cervicogenic dizziness: a comprehensive review of its aetiopathology and management. European Archives of Oto-RhinoLaryngology. 275(10): 2 421-2433.

31. Greenbaum T, Dvir Z, Reiter S, Winocur E. (2017) Cervical flexion-rotation test and physiological range of motion - A comparative study of patients with myogenic temporomandibular disorder versus healthy subjects. Musculoskeletal Science and Practice. 27: 7-13. 
32. Luedtke K, Starke W, May A. (2017) Musculoskeletal dysfunction in migraine patients. Cephalalgia. 38(5): 865-875.

33. Mutlu B, Serbetcioglu B. (2013) Discussion of the dizziness handicap inventory. Journal of Vestibular Research. 23(6): 271-277.

34. Reid SA, Callister R, Katekar MG, Treleaven JM. (2017) Utility of a brief assessment tool developed from the Dizziness Handicap Inventory to screen for Cervicogenic dizziness: A case control study. Musculoskeletal Science and Practice. 30: 42-48.

35. Ohrbach R, Larsson P, List T. (2008) The jaw functional limitation scale: development, reliability, and validity of 8-item and 20-item versions. Journal of Orofacial Pain. 22(3): 219-230.

36. Schiffman E, Ohrbach R, Truelove E, et al. (2014) Diagnostic Criteria for Temporomandibular Disorders (DC/TMD) for Clinical and Research Applications: Recommendations of the International RDC/TMD Consortium Network and Orofacial Pain Special Interest Group $\dagger$. Journal of Oral \& Facial Pain and Headache. 28(1): 6-27.

37. De Hertogh WJ, Vaes PH, Vijverman V, De Cordt A, Duquet W. (2007) The clinical examination of neck pain patients: The validity of a group of tests. Manual Therapy. 12(1): 50-55.

38. Piekartz H, Schwiddessen J, Reineke L, et al. (2020) International consensus on the most useful assessments used by physical therapists to evaluate patients with temporomandibular disorders: A Delphi study. Journal of Oral Rehabilitation. 47(6): 685-702.

39. Luedtke K, Boissonnault W, Caspersen N, et al. (2016) International consensus on the most useful physical examination tests used by physiotherapists for patients with headache: A Delphi study. Manual Therapy. 23: 1724.
40. Von Piekartz H, Pudelko A, Danzeisen M, Hall T, Ballenberger N. (2016) Do subjects with acute/subacute temporomandibular disorder have associated cervical impairments: A cross-sectional study. Manual Therapy. 26: n208-215.

41. Michiels S, Van de Heyning P, Truijen S, De Hertogh W. (2015) Diagnostic Value of Clinical Cervical Spine Tests in Patients with Cervicogenic Somatic Tinnitus. Physical Therapy. 95(11): 1529-1535.

42. Finan PH, Goodin BR, Smith MT. (2013) The Association of Sleep and Pain: An Update and a Path Forward. The Journal of Pain. 14(12): 1539-1552.

43. Naugle KM, Fillingim RB, Riley JL. (2012) A MetaAnalytic Review of the Hypoalgesic Effects of Exercise. The Journal of Pain. 13(12): 1139-1150.

44. Ornello R, Ripa P, Pistoia F, et al. (2015) Migraine and body mass index categories: a systematic review and meta-analysis of observational studies. The Journal of Headache and Pain. 16(1).

45. LaRowe LR, Ditre JW. (2020) Pain, nicotine, and tobacco smoking: current state of the science. Pain. 161(8): 1688-1693.

46. Hirshkowitz M, Whiton K, Albert SM, et al. (2015) National Sleep Foundation's sleep time duration recommendations: methodology and results summary. Sleep Health. 1(1): 40-43.

47. Department of Health and Social Care. (2019) UK Chief Medical Officers' Physical Activity Guidelines.

48. Robblee J, Starling AJ. 2019) SEEDS for success: Lifestyle management in migraine. Cleveland Clinic Journal of Medicine. 86(11): 741-749.

49. Wideman TH, Edwards RR, Walton DM, Martel MO, Hudon A, Seminowicz DA. (2019) The Multimodal Assessment Model of Pain. The Clinical Journal of Pain. 35(3): 212-221. 